\title{
THE STUDY OF THE HYDROSTATIC PRESSURE EFFECT ON THE THERMODYNAMIC PROPERTIES OF THE ROCHELLE SALT NaKC $\mathrm{H}_{4} \mathrm{O}_{6} \cdot 4 \mathrm{H}_{2} \mathrm{O}$
}

\author{
R. R. Levitskii ${ }^{1}$, A. P. Moina ${ }^{1}$, A. Ya. Andrusyk ${ }^{1}$, A. G. Slivka², V. M. Kedyulich ${ }^{2}$ \\ ${ }^{1}$ Institute for Condensed Matter Physics of the National Academy of Sciences of Ukraine, \\ 1, Sventsitsky St., Lviv, UA-79011, Ukraine \\ ${ }^{2}$ Uzhgorod National University, 46, Pidhirna St., Uzhgorod, UA-88000, Ukraine
}

(Received December 24, 2007; received in final form March 27, 2008)

\begin{abstract}
The effect of hydrostatic pressure on thermodynamic properties of Rs was theoretically considered within the framework of the Mitsui model adjusted by taking into account piezoelectric interaction with the shear strain $\varepsilon_{4}$. The approach, according to which theory model parameters are linearly dependent on hydrostatic pressure, was proposed for consideration. The calculations were carried out in molecular field approximation. Theoretical results for thermodynamic characteristics were compared to experimental data. Basing on theoretical study, the explanation for discrepancies in experimental data derived by different researchers for static dielectric permittivity of a free crystal was proposed. The increase of saturation polarization due to application of hydrostatic pressure is predicted.
\end{abstract}

Key words: Rochelle salt, hydrostatic pressure effect, molecular field approximation.

PACS number(s): 64.60.Cn, 77.22.-d, 77.80.Bh

\section{INTRODUCTION}

Important information about ferroelectric crystals can be obtained from the high pressure studies. Being the only means to continuously vary the geometric parameters of the crystal lattice, hydrostatic pressure allows to explore to what extent transition temperature and dielectric response of hydrogen bonded crystals depend on the geometric parameters of hydrogen bonds. From the theoretical point of view, the pressure studies provide a deeper insight into the statistical mechanics of the models used to describe the particular ferroelectric crystals.

Consistent theories (e.g. [1] for the $\mathrm{KH}_{2} \mathrm{PO}_{4}$ type crystals) approach the problem of the study of pressure effect on physical properties from the microscopical standpoint, considering contributions of piezoelectric and electrostrictional couplings of the pseudospin variables with acoustic and optical phonons. Within a linear approximation those contributions for the hydrostatic pressure case can be reduced to additional internal fields, linear in pressure-induced lattice strains. There are also contributions arising from the pressure variation of the dipole moments, of the local geometry of the system (not determined by the macroscopic lattice strains) under hydrostatic pressure effect that should be taken into account.

In a particular case of the $\mathrm{KH}_{2} \mathrm{PO}_{4}$ family ferroelectrics under the hydrostatic pressure or the uniaxial $p=-\sigma_{3}$ stress the changes in the long-range interaction parameters due to the pressure variation of the dipole moments as well as the linear in lattice strains fields due to the electrostrictional coupling with the diagonal components of the strain tensor were taken into account [2]. This work also considered the pressure variation of the short-range configurational interactions between pseudospins, attributing it solely to the pressure variation of the hydrogen bonds geometry, namely, to the $\mathrm{H}$-site distance $\delta$. The calculations of [2] revealed a universal linear $T_{\mathrm{C}}\left(T_{\mathrm{N}}\right)$ vs $\delta$ behaviour in several deuterated ferroelectric and antiferroelectric $M e \mathrm{D}_{2} \mathrm{XO}_{4}$ crystals $(M e$ $=\mathrm{K}, \mathrm{Rb}, \mathrm{ND}_{4}, X=\mathrm{P}, \mathrm{As}$ ), similar to the experimentally found one [3] in $\mathrm{KH}_{2} \mathrm{PO}_{4}, \mathrm{KD}_{2} \mathrm{PO}_{4}, \mathrm{NH}_{4} \mathrm{H}_{2} \mathrm{PO}_{4}$, and $\mathrm{ND}_{4} \mathrm{D}_{2} \mathrm{PO}_{4}$.

Within the same approach there were also considered the cases of external stresses that do lower the symmetry of the $\mathrm{KH}_{2} \mathrm{PO}_{4}$ type crystals: a shear stress $\sigma_{6}$, reducing the crystal symmetry in the paraelectric phase to that of the ferroelectric phase, and a symmetrized stress $\sigma_{1}-\sigma_{2}$, breaking the equivalence of the hydrogen bonds oriented along the $a$ and $b$ axes. In the first case, the piezoelectric internal field linear in the shear strain $\varepsilon_{6}$ as well as splitting of the short-range configurational interactions due to lowering the crystal symmetry were taken into account [4]. In the second case, the theory also included the splitting as well as the internal fields due to electrostrictional coupling with the strains $\varepsilon_{1}$ and $\varepsilon_{2}[5,6]$. A possibility was revealed that a new stress-induced monoclinic phase can exist in the $\mathrm{KH}_{2} \mathrm{PO}_{4}$ type crystals.

Though sophisticated the microscopic approach to modelling the deformed crystal is, for $\mathrm{KH}_{2} \mathrm{PO}_{4}$ type ferroelectrics one can easily show that the used dependencies of the model parameters on lattice strains and H-site distance can be effectively replaced (in case of hydrostatic pressure) with a phenomenological linear dependence of the parameters on the applied hydrostatic pressure. Phenomenological linear dependencies of theory model parameters on applied hydrostatic pressure can be used by analogy for other types of crystals.

Though such an approach gives little insight into the fundamental microscopic physics involved into the behaviour of the deformed crystal, it allows to describe the observed pressure dependencies of the physical characteristics of the crystals and explore the used model more thoroughly. Such an approach has been used for the case of $\mathrm{CsH}_{2} \mathrm{PO}_{4}$ and $\mathrm{CsD}_{2} \mathrm{PO}_{4}$ crystals, where it 
is known $[7,8]$ that particular pressure makes the character of the ordering change from ferroelectric to antiferroelectric. It was assumed $[9,10]$ that the interaction constant changes its sign at this pressure. By virtue of the proper choice of the model parameters a fair description of experimental data for the pressure variation of the transition temperatures and static dielectric permittivity of $\mathrm{CsH}_{2} \mathrm{PO}_{4}, \mathrm{CsD}_{2} \mathrm{PO}_{4}$, and $\mathrm{RbD}_{2} \mathrm{PO}_{4}$ crystals was obtained [10].

Rochelle salt is a particular case, where, on the one hand, an order-disorder model (Mitsui model) does exist and it well describes the complicated system behaviour, including two phase transitions and an intermediate ferroelectric phase, on the other hand, it is still not quite clear with what atoms should the model dipoles be associated. It is, therefore, rather problematic to attempt to perform any consistent microscopic description of the crystal behaviour under the external pressure. In this case, the half-phenomenological approach to this problem, applied in this work, where the parameters of the model are assumed to be linear functions of external pressure, can be deemed acceptable.

Experimentally, the dependencies of the transition temperatures, spontaneous polarization and the static dielectric permittivity of Rochelle salt on hydrostatic pressure were determined [11-14]. Studies [14,15] were devoted, among other issues, to the uniaxial stresses influence on the transition temperatures and dielectric permittivity of this crystal. No theoretical calculations of these dependencies are available to our best knowledge. The purpose of this work is to propose an approach to theoretical description of hydrostatic pressure effect on physical properties of Rochelle salt based on the previous results.

We shall use the modified Mitsui model [16], which takes into account the piezoelectric coupling with the spontaneous strain $\varepsilon_{4}$. The model is free from the drawbacks of the conventional Mitsui model and allows a quantitative description $[17,18]$ of the system dynamics within the $1 \mathrm{kHz}-1 \mathrm{THz}$ frequency range, of which the conventional model is not capable. Within the proposed approach the model parameters will be assumed to be linear functions of external pressure. On the basis of this assumption the pressure dependencies of the transition temperatures, dielectric, piezoelectric, and elastic characteristics of Rochelle salt crystals will be calculated and compared with available experimental data.

\section{THERMODYNAMIC CHARACTERISTICS OF THE MITSUI MODEL THAT TAKES INTO ACCOUNT THE PIEZOELECTRIC INTERACTION UNDER HYDROSTATIC PRESSURE EFFECT}

First we consider a two-sublattice piezoelectric orderdisorder type system with an asymmetric double-well potential that is not effected by external pressure. Systems polarization is directed along $x$-axis and appears due to ordering of the opposite sublattice structure elements in different equilibrium states. Piezoelectric interactions are regarded to be considerable and the shear strain $\varepsilon_{4}=\varepsilon_{y z}$ is considered to influence the energy of equilibrium states. The model states that the phase transitions are caused by the motion of certain dipoles in asymmetric double-well potentials with two different constants for the interaction between dipoles of the same sublattice and of different sublattices. Such system is adequate for Rochelle salt. Hamiltonian of this system, proposed in work [16], is a modified Mitsui Hamiltonian:

$$
H=\frac{N}{2} v c_{44}^{\mathrm{E} 0} \varepsilon_{4}^{2}-N v e_{14}^{0} E_{1} \varepsilon_{4}-\frac{N}{2} v \chi_{11}^{\varepsilon 0} E_{1}^{2}-\frac{1}{2} \sum_{\substack{q, f \\ q^{\prime} f^{\prime}}} R_{q q^{\prime}}^{f f^{\prime}} S_{q f}^{z} S_{q^{\prime} f^{\prime}}^{z}-\sum_{q f}\left(\Delta_{f} S_{q f}^{z}-2 \psi_{4} \varepsilon_{4}+\mu E_{1}\right) S_{q f}^{z}
$$

The three first terms in (1.1) represent the elastic, piezoelectric, and electric energies attributed to a host lattice in which potential the quasispin move (with the "seed" elastic constant $c_{44}^{\mathrm{E} 0}$, the coefficient of piezoelectric stress $e_{14}^{0}$, and dielectric susceptibility $\left.\chi_{11}^{\varepsilon 0}\right) ; v$ is a half of the unit cell volume. In the fourth term in Eq. (1.1), indexes $f, f^{\prime}$ number sublattices, $q, q^{\prime}$ number crystal units, each of them consisting of two quasispins, $R_{q q^{\prime}}^{11}=R_{q q^{\prime}}^{22}=J_{q q^{\prime}}, R_{q q^{\prime}}^{12}=R_{q q^{\prime}}^{21}=K_{q q^{\prime}}$ are the potentials of interaction between quasispins belonging to the same and to different sublattices, respectively. The fifth term consists of three parts. The first part is energy associated with the asymmetry of double-well potential and $\Delta_{f}$ represents the value of asymmetry $\left(\Delta_{1}=-\Delta_{2}=\Delta\right)$. The second part is energy associated with the interaction of dipoles with an additional internal field generated by piezoelectric coupling with the shear strain $\varepsilon_{4} ; \psi_{4}$ is the intensity of this interaction. The third part is system's energy associated with the interaction of dipoles with external electric field and $\mu$ is the effective dipole moment of unit cell.

Below we present results of work [16] for thermodynamic characteristics of this model, derived within molecular field approximation. Hamiltonian and free energy of two quasispins in this approximation are the following:

$$
\begin{aligned}
H & =\frac{N}{2} v c_{44}^{\mathrm{E} 0} \varepsilon_{4}^{2}-N v e_{14}^{0} E_{1} \varepsilon_{4}-\frac{N}{2} v \chi_{11}^{\varepsilon 0} E_{1}^{2} \\
& +k_{\mathrm{B}} N \frac{1}{2}\left[\tilde{R}^{+} \xi^{2}+\tilde{R}^{-} \sigma^{2}\right]-k_{\mathrm{B}} \sum_{q f} \tilde{\varepsilon}_{f} S_{q f}^{z},
\end{aligned}
$$




$$
\begin{aligned}
& f(4)=\frac{1}{2} \tilde{v} c_{44}^{\mathrm{E} 0} \varepsilon_{4}{ }^{2}-\tilde{v} e_{14}^{0} \varepsilon_{4} E_{1}-\frac{1}{2} \tilde{v} \chi_{11}^{\varepsilon 0} E_{1}{ }^{2} \\
& +\frac{1}{2}\left[\tilde{R}^{+} \xi^{2}+\tilde{R}^{-} \sigma^{2}\right]-T \sum_{f} \ln \left(2 \cosh \frac{\tilde{\varepsilon}_{f}}{2 T}\right),
\end{aligned}
$$

where $\tilde{v}=\frac{v}{k_{\mathrm{B}}}$ and the following notations are used:

$$
\begin{aligned}
& \tilde{\varepsilon}_{1}=\tilde{R}^{+} \xi+\tilde{R}^{-} \sigma+\tilde{\Delta}-2 \tilde{\psi}_{4} \varepsilon_{4}+\tilde{\mu} E_{1} \\
& \tilde{\varepsilon}_{2}=\tilde{R}^{+} \xi-\tilde{R}^{-} \sigma-\tilde{\Delta}-2 \tilde{\psi}_{4} \varepsilon_{4}+\tilde{\mu} E_{1} .
\end{aligned}
$$

Here

$$
\begin{aligned}
& \tilde{\Delta}=\Delta / k_{\mathrm{B}}, \quad \tilde{\psi}_{4}=\psi_{4} / k_{\mathrm{B}}, \quad \tilde{\mu}=\mu / k_{\mathrm{B}}, \\
& \tilde{R}^{ \pm}=\frac{\tilde{J}_{0} \pm \tilde{K}_{0}}{2}, \quad \tilde{J}_{0}=\frac{J_{0}}{k_{\mathrm{B}}}, \quad \tilde{K}_{0}=\frac{K_{0}}{k_{\mathrm{B}}} . \\
& J_{0}=\sum_{q^{\prime}} J_{q q^{\prime}}=\sum_{q^{\prime}} R_{q q^{\prime}}^{11}=\sum_{q^{\prime}} R_{q q^{\prime}}^{22}, \\
& K_{0}=\sum_{q^{\prime}} K_{q q^{\prime}}=\sum_{q^{\prime}} R_{q q^{\prime}}^{12}=\sum_{q^{\prime}} R_{q q^{\prime}}^{21} .
\end{aligned}
$$

Variational parameters $\xi$ and $\sigma$ ( $\xi$ is ferroelectric ordering parameter) are defined from the following set of equations where $T, E_{1}, \varepsilon_{4}$ are known:

$$
\left\{\begin{array}{l}
\xi=\frac{1}{2}\left[\tanh \frac{\tilde{\varepsilon}_{1}}{2 T}+\tanh \frac{\tilde{\varepsilon}_{2}}{2 T}\right], \\
\sigma=\frac{1}{2}\left[\tanh \frac{\tilde{\varepsilon}_{1}}{2 T}-\tanh \frac{\tilde{\varepsilon}_{2}}{2 T}\right] .
\end{array}\right.
$$

From thermodynamic equilibrium conditions

$$
\frac{1}{\tilde{v}}\left(\frac{\partial f(4)}{\partial \varepsilon_{4}}\right)_{\mathrm{E}_{1}, \mathrm{~T}}=\sigma_{4}, \quad \frac{1}{\tilde{v}}\left(\frac{\partial f(4)}{\partial E_{1}}\right)_{\varepsilon_{4}, \mathrm{~T}}=-P_{1}
$$

the shear stress $\sigma_{4}$ and polarization $P_{1}$ was derived:

$$
\begin{aligned}
\sigma_{4} & =c_{44}^{\mathrm{E} 0} \varepsilon_{4}-e_{14}^{0} E_{1}+\frac{2 \tilde{\psi}_{4}}{\tilde{v}} \xi, \\
P_{1} & =e_{14}^{0} \varepsilon_{4}+\chi_{11}^{\varepsilon 0} E_{1}+\frac{\tilde{\mu}}{\tilde{v}} \xi .
\end{aligned}
$$

If an independent variable is not a shear strain but a shear stress (the case with $\sigma_{4}=0$ is considered in this work), then while solving equations system (1.5) local fields $\tilde{\varepsilon}_{1}, \tilde{\varepsilon}_{2}$ should be expressed not in terms of $\varepsilon_{4}$ but in terms of $\sigma_{4}$. The first equation of (1.6) yields

$$
\varepsilon_{4}=\frac{\sigma_{4}}{c_{44}^{\mathrm{E} 0}}+\frac{e_{14}^{0}}{c_{44}^{\mathrm{E} 0}} E_{1}-\frac{2 \tilde{\psi}_{4}}{\tilde{v} c_{44}^{\mathrm{E} 0}} \xi .
$$

Now it is possible to rewrite local fields in the following form:

$$
\begin{aligned}
& \tilde{\varepsilon}_{1}=\tilde{R}^{\prime+} \xi+\tilde{R}^{-} \sigma+\tilde{\Delta}+\tilde{\delta} \\
& \tilde{\varepsilon}_{2}=\tilde{R}^{\prime+} \xi-\tilde{R}^{-} \sigma-\tilde{\Delta}+\tilde{\delta} \\
& \tilde{\delta}=-\frac{2 \tilde{\psi}_{4}}{c_{44}^{\mathrm{E} 0}} \sigma_{4}+\tilde{\mu}^{\prime} E_{1},
\end{aligned}
$$

where $\tilde{R}^{\prime+}$ and $\tilde{\mu}^{\prime}$ are the following:

$$
\begin{aligned}
& \tilde{R}^{\prime+}=\tilde{R}^{+}+\frac{4 \tilde{\psi}_{4}^{2}}{\tilde{v} c_{44}^{\mathrm{E} 0}} \\
& \tilde{\mu}^{\prime}=\tilde{\mu}-\frac{2 \tilde{\psi}_{4} e_{14}^{0}}{c_{44}^{\mathrm{E} 0}} .
\end{aligned}
$$

By differentiating expressions (1.6) the clamped static dielectric susceptibility along a-axis $\chi_{11}^{\varepsilon}=\left(\frac{\partial P_{1}}{\partial E_{1}}\right)_{\varepsilon_{4}}$, the coefficient of the piezoelectric stress $e_{14}=\left(\frac{\partial P_{1}}{\partial \varepsilon_{4}}\right)_{\mathrm{E}_{1}}$ and the elastic constant at constant field $c_{44}^{\mathrm{E}}=\left(\frac{\partial \sigma_{4}}{\partial \varepsilon_{4}}\right)_{\mathrm{E}_{1}}$ were derived:

$$
\begin{gathered}
\chi_{11}^{\varepsilon}=\chi_{11}^{\varepsilon 0}+\frac{\tilde{\mu}^{2}}{\tilde{v}} f\left(T, \sigma_{4}, E_{1}\right), \\
e_{14}=e_{14}^{0}-\frac{2 \tilde{\psi}_{4} \tilde{\mu}^{\prime}}{\tilde{v}} f\left(T, \sigma_{4}, E_{1}\right), \\
c_{44}^{\mathrm{E}}=c_{44}^{\mathrm{E} 0}-\frac{4 \tilde{\psi}_{4}^{2}}{\tilde{v}} f\left(T, \sigma_{4}, E_{1}\right),
\end{gathered}
$$

where

$$
\begin{aligned}
& f\left(T, \sigma_{4}, E_{1}\right)=\frac{e_{1} A_{22}-e_{2} A_{12}}{A_{11} A_{22}-A_{12} A_{21}}, \\
& A_{11}=1-\tilde{R}^{+} e_{1}, \quad A_{12}=-\tilde{R}^{-} e_{2}, \\
& A_{21}=-\tilde{R}^{+} e_{2}, \quad A_{22}=1-\tilde{R}^{-} e_{1}, \\
& e_{1}=-\frac{\xi^{2}+\sigma^{2}}{2 T}, \quad e_{2}=-\frac{\xi \sigma}{T} .
\end{aligned}
$$

The following are the other dielectric, piezoelectric and elastic characteristics which can be expressed through those above:

the constant of piezoelectric stress

$$
h_{14}=-\left(\frac{\partial E_{1}}{\partial \varepsilon_{4}}\right)_{P_{1}}=\frac{e_{14}}{\chi_{11}^{\varepsilon}}
$$

the elastic constant at constant polarization:

$$
c_{44}^{\mathrm{P}}=\left(\frac{\partial \sigma_{4}}{\partial \varepsilon_{4}}\right)_{P_{1}}=c_{44}^{\mathrm{E}}+e_{14} h_{14} ;
$$

the coefficient of the piezoelectric strain:

$$
d_{14}=\left(\frac{\partial P_{1}}{\partial \sigma_{4}}\right)_{\mathrm{E}_{1}}=\frac{e_{14}}{c_{44}^{\mathrm{E}}}
$$

the constant of piezoelectric strain

$$
g_{14}=-\left(\frac{\partial E_{1}}{\partial \sigma_{4}}\right)_{P_{1}}=\frac{h_{14}}{c_{44}^{\mathrm{P}}}
$$

the elastic compliance at constant field:

$$
s_{44}^{\mathrm{E}}=\left(\frac{\partial \varepsilon_{4}}{\partial \sigma_{4}}\right)_{\mathrm{E}_{1}}=\frac{1}{c_{44}^{\mathrm{E}}}
$$


the dielectric susceptibility of a free crystal:

$$
\chi_{11}^{\sigma}=\left(\frac{\partial P_{1}}{\partial E_{1}}\right)_{\sigma_{4}}=\chi_{11}^{\varepsilon}+e_{14} d_{14} .
$$

The molar entropy of the quasispin subsystem of the Rochelle salt is

$$
\begin{aligned}
S_{4} & =-R \frac{1}{2 m}\left(\frac{\partial f(4)}{\partial T}\right)_{\mathrm{E}_{1}, \varepsilon_{4}} \\
& =\frac{R}{2 m}\left\{\sum_{f}\left[\ln \left(2 \cosh \frac{\tilde{\varepsilon}_{f}}{2 T}\right)-\frac{\tilde{\varepsilon}_{f}}{2 T} \tanh \frac{\tilde{\varepsilon}_{f}}{2 T}\right]\right\},
\end{aligned}
$$

where $R$ is universal gas constant, $m$ is the number of molecules that forms one quasispin (dipole). In the case of Rochelle salt $m=1$.

We calculate the molar specific heat of the quasispin subsystem at constant stress and electric field by numerical differentiation of entropy:

$$
\Delta C_{4}^{\sigma \mathrm{E}}=T\left(\frac{\partial S_{4}}{\partial T}\right)_{\sigma_{4}, \mathrm{E}_{1}} .
$$

Studying hydrostatic pressure effect on the physical characteristics of Rs we supposed that theory model parameters $\tilde{J}_{0}, \tilde{K}_{0}, \tilde{\Delta}$ linearly depend on applied hydrostatic pressure:

$$
\begin{aligned}
\tilde{J}_{0} & =\tilde{J}_{0}(0)(1+\alpha p) \\
\tilde{K}_{0} & =\tilde{K}_{0}(0)(1+\varkappa \alpha p) \\
\tilde{\Delta} & =\tilde{\Delta}(0)(1+\beta p),
\end{aligned}
$$

where $\tilde{J}_{0}(0), \tilde{K}_{0}(0), \tilde{\Delta}(0)$ are the theory model parameters with zero hydrostatic pressure, $p$ is the hydrostatic pressure, $\alpha, \varkappa, \beta$ unknown theory parameters. We consider parameter $\tilde{\psi}_{4}$ independent of pressure as within proposed linear approximation its dependence on pressure would just result in the appearance of high order terms. By the same reason we consider "seed" parameters $c_{44}^{\mathrm{E} 0}, e_{14}^{0}, \chi_{11}^{\varepsilon 0}$ to be also independent from pressure. Dependence of effective dipole moment on hydrostatic pressure will be discussed below.

Thus, the study of hydrostatic pressure effect on physical characteristics of Rs basing on this approach reduces to the choice of additional theory parameters $\alpha, \varkappa, \beta$.

\section{NUMERICAL ANALYSIS. COMPARING THEORETICAL RESULTS WITH EXPERIMENTAL ONES. DISCUSSION OF THE DERIVED RESULTS}

Experimental data shows that Rochelle Salt preserves its general physical properties at hydrostatic pressure up to 25 kbar: it has two second order phase transitions, where at the lower Curie point the occurring transition is from nonpolar phase to ferroelectric and at the higher Curie point the transition is reverse.

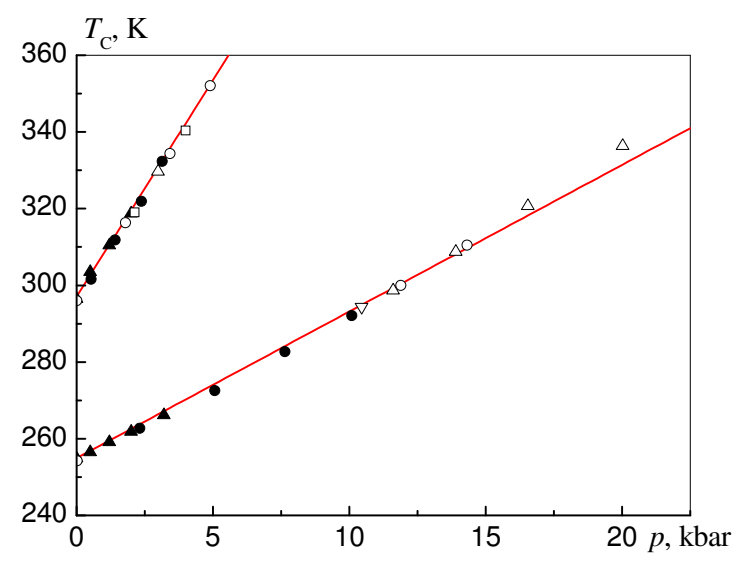

Fig. 1. Experimentally measured dependencies of the phase transition temperatures of Rochelle salt on hydrostatic pressure. The points $\boldsymbol{\Delta}$ represent data of the work [15], • $-[11], \circ, \triangle, \square, \nabla-[12]$. The figure presents linear approximation of the experimental data too.

Experimental dependencies of phase transition temperatures on the hydrostatic pressures are presented on figure 1 . We found parameters $\alpha, \varkappa$ and $\beta$ under condition of agreement of theory results and experiment data for dependency $T_{\mathrm{C}}(p)$. Calculations require some theory parameters adequate to description of the Rochelle salt that does not undergo hydrostatic pressures effect. They were derived previously in work [16]:

$$
\begin{aligned}
& \tilde{J}_{0}(0)=797.36 \mathrm{~K} \\
& \tilde{K}_{0}(0)=1468.83 \mathrm{~K} \\
& \tilde{\Delta}(0)=737.33 \mathrm{~K} \\
& \tilde{\psi}_{4}=-760 \mathrm{~K} \\
& c_{44}^{E 0}=1.28 \cdot 10^{10} \mathrm{~N} / \mathrm{m}^{2} \\
& e_{14}^{0}=3.336 \cdot 10^{-2} \mathrm{C} / \mathrm{m}^{2} \\
& \chi_{11}^{\varepsilon 0}=0.318 \\
& \mu(T)=a+k(T-297) \\
& a=8.41 \cdot 10^{-30} \mathrm{C} \cdot \mathrm{m} \\
& k=-0.022 \cdot 10^{-30}(\mathrm{C} \cdot \mathrm{m}) / \mathrm{K} .
\end{aligned}
$$

Also, we need to know the elementary cell volume at defined hydrostatic pressure and temperature for previous calculations. Basing on the result for elastic compliances derived in work [19] at $T=307 \mathrm{~K}$ and on the result for elementary cell volume in absence of hydrostatic pressure derived in work [20] we present dependence of the elementary cell volume on hydrostatic pressure at $T=307 \mathrm{~K}$ in the form:

$$
v(p)=529.84 \cdot 10^{-30}(1-0.00445 p)
$$

where pressure is measured in kilobars.

Work [21] showed that Rochelle salt elastic constants $c_{i j}^{\mathrm{E}}$ are almost independent from the temperature. This result along with the very slight dependence of elementary volume cell on temperature in absence of hydrostatic pressure [20] allow the conclusion that dependence of elementary volume cell on the temperature is small at any 
pressure and may be neglected. Therefore, for further calculations we will use (2.2) at any temperature.

Studies of the behaviour dependence of our system on pressure at different parameters sets $\alpha, \varkappa$ and $\beta$ show that some parameters sets result in phase transitions sequence other than our system should undergo at the given pressure. To exclude such parameters sets from studies, we used phase diagram of the Mitsui model plotted in work [22]. Specifically, we consider only the parameters sets presented by the point, which in phase diagram corresponds to the Rochelle salt at every specific pressure $(<25 \mathrm{kbar})$ and lies within such a region where only two second order phase transitions exist.

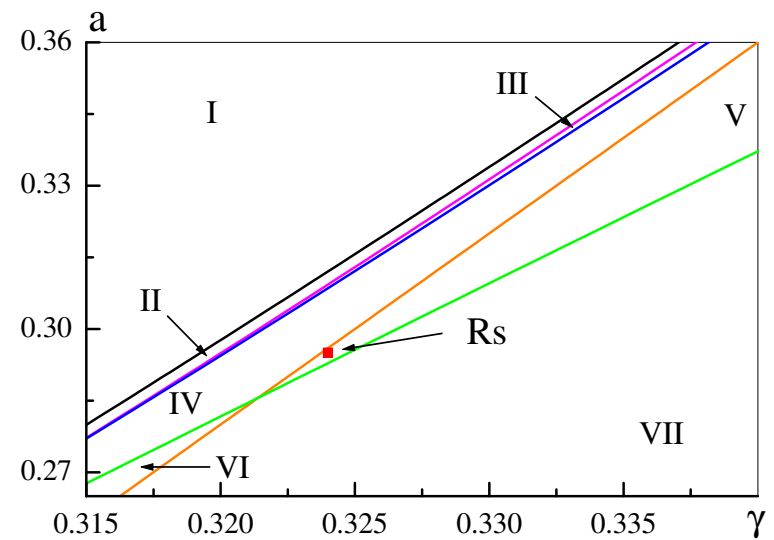

Fig. 2. Phase diagram fragment of the Mitsui model, presented in variables $a=-\frac{\tilde{R}^{-}}{R^{\prime+}}$ and $\gamma=\frac{\tilde{\Delta}}{2 R^{\prime+}}$. The region with two second order phase transition is shown in phase diagram. The point corresponding to the Rochelle salt theory parameters in absence of hydrostatic pressure is presented in this region.

Fragment of the phase diagram is shown in figure 2 . The following regions are presented there:

I - ground state is ordered; system undergos second order p.t. (phase transition) from ordered to disordered state;

II - ground state is ordered; two low temperature p.t. of first order, and one p.t. of second order;

III - ground state is ordered; first order p.t. (from ordered to disordered state), second order p.t. (from disordered to ordered state), first order p.t. within the ferroelectric phase and second order p.t. to the disordered state;

IV - ground state is ordered; one low temperature p.t. of first order and two second order p.t.;

$\mathrm{V}$ - ground state is disordered; two second order p.t.; VI - ground state is ordered; one first order p.t.;

VII - p.t. are absent, disordered state is stable.

Hence, parameters point that represents Rochelle salt at every specific pressure $(<25 \mathrm{kbar})$ should lie within the region $\mathrm{V}$.

Besides that, calculated dependencies of the phase transition temperatures on the hydrostatic pressure must correspond to experimental data. Analysis shows that only positive parameters $\alpha, \varkappa$ and $\beta$ satisfy the conditions presented above. We derived 9 such parameters sets that satisfy the necessary conditions.
1) $\alpha=0.024119, \quad \beta=0.030254140625, \quad \varkappa=1.26$;

2) $\alpha=0.023801, \quad \beta=0.030937265625, \quad \varkappa=1.30$;

3) $\alpha=0.023492, \quad \beta=0.031603564453, \quad \varkappa=1.34$;

4) $\alpha=0.023043, \quad \beta=0.032570839840, \quad \varkappa=1.40$;

5) $\alpha=0.022484, \quad \beta=0.034355771484, \quad \varkappa=1.50$;

6) $\alpha=0.021320, \quad \beta=0.037444091797, \quad \varkappa=1.70$;

7) $\alpha=0.019741, \quad \beta=0.041424570313, \quad \varkappa=2.00$;

8) $\alpha=0.017762, \quad \beta=0.047430888670, \quad \varkappa=2.50$;

9) $\alpha=0.011458, \quad \beta=0.063464990230, \quad \varkappa=5.00$.

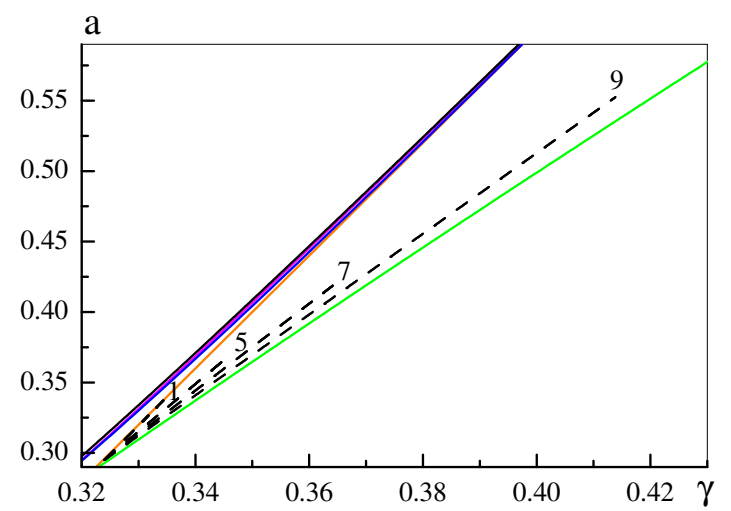

Fig. 3. Phase diagram fragment of the Mitsui model. Dashed lines show the trajectories of the Rochelle salt parameters points at variation of the hydrostatic pressure form 0 kbar to $25 \mathrm{kbar}$. Parameters points correspond to sets 1, 5, 7,9 presented in $(2.3)$.

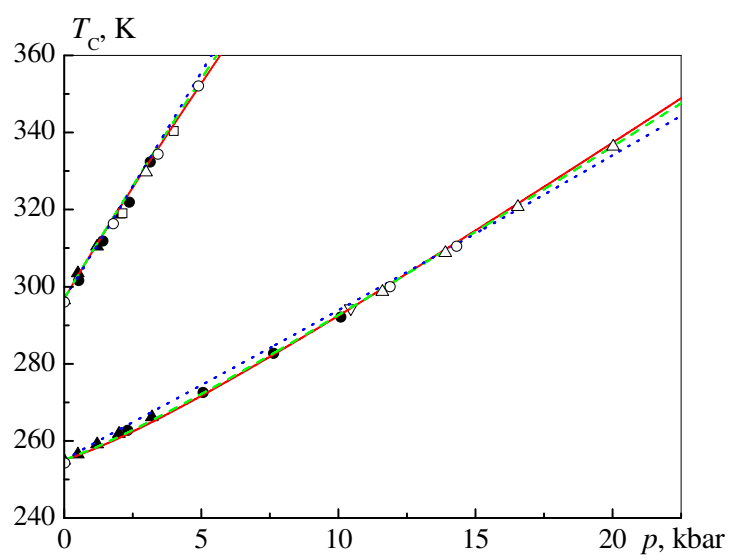

Fig. 4. Results of theoretical calculations for $T_{\mathrm{C}}(p)$ dependence along with experimental data. Solid line denotes the results of calculations derived for parameters set 1, dashed 7 , dotted -9 (see $(2.3)$ ). Experimental points are the same as in figure 1.

Figure 3 shows lines in the phase diagram that represent the path of the point correspondent to Rochelle salt at variation of the hydrostatic pressure form $0 \mathrm{kbar}$ to $25 \mathrm{kbar}$. These lines were obtained for parameters sets 1 , $5,7,9$. In figure 4 the results of theoretical calculations for dependence $T_{\mathrm{C}}(p)$ derived for parameters sets $1,7,9$ are presented along with experimental data. 


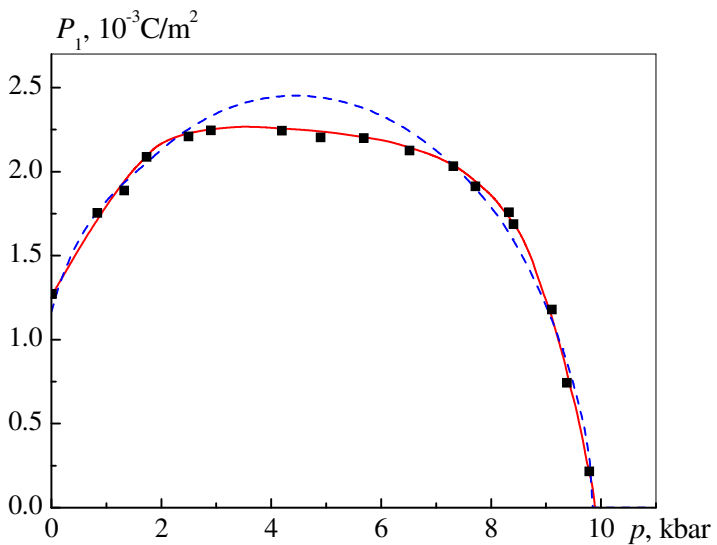

Fig. 5. Dependence of the spontaneous polarization on the hydrostatic pressure at $T=292 \mathrm{~K}$. Points present experimental data of work [12]. Solid line presents the approximation of the experimental data, dashed line presents the results of theoretical calculation with parameters set 7 and effective dipole moment taken according to (2.4).

From all of the derived parameters sets we will choose one set under condition of minimum mean square deviation of theoretical results for $T_{\mathrm{C}}(p)$ from the experiment data. Parameters set 7 satisfies this condition. We will consider this parameters set as the most appropriate to the description of physical properties of the Rochelle salt and all further calculations will be carried out with this parameter set. However, we cannot be quite confident that the deduced inference is true. In order to define parameters set, which is the most appropriate for Rochelle salt, with greater confidence, we need to verify agreement of theoretical results with experimental data for other physical characteristics that do not require new adjustable parameters for calculations. However, we have no such experimental data.

Now we will find out a dependence of the effective dipole moment on the hydrostatic pressure. For this purpose we will use experimental data of work [12] that is presented in figure 5. This figure shows a dependence of polarization on pressure at room temperature $(T=292 \mathrm{~K})$. We will derive effective dipole moment under condition of coincidence of the theoretical results and approximation of the experimental data for the mentioned above dependence. Figure 6 shows dependencies of effective dipole moment on pressure at $T=292 \mathrm{~K}$ that were calculated in such a way.

We propose to approximate the derived dependence of the effective dipole moment on hydrostatic pressure at $T=292 \mathrm{~K}$ by the following smooth function:

$$
\begin{aligned}
& \mu_{0}=\left(0.33445 \cdot p^{2}-1.40390 \cdot p+8.52\right) \cdot 10^{-30} \mathrm{Cm} \quad(p \leq 2 \mathrm{kbar}) \\
& \mu_{0}=(-0.06610 \cdot p+7.18220) \cdot 10^{-30} \mathrm{Cm} \quad(2 \mathrm{kbar}<p \leq 8 \mathrm{kbar}) \\
& \mu_{0}=\left(-0.2703 \cdot p^{2}+4.2588 \cdot p-10.117\right) \cdot 10^{-30} \mathrm{Cm} \quad(8 \mathrm{kbar}<p \leq 10 \mathrm{kbar})
\end{aligned}
$$

where we accounted that according to (2.1) at zero pressure and $T=292 \mathrm{~K}$ effective dipole moment is equal to $8.52 \cdot 10^{-30} \mathrm{Cm}$. It is clear that the derived dependence of effective dipole moment is valid for not too high pressures, in our case for $p \leq 10 \mathrm{kbar}$. The result of the approximation is presented in figure 6 .

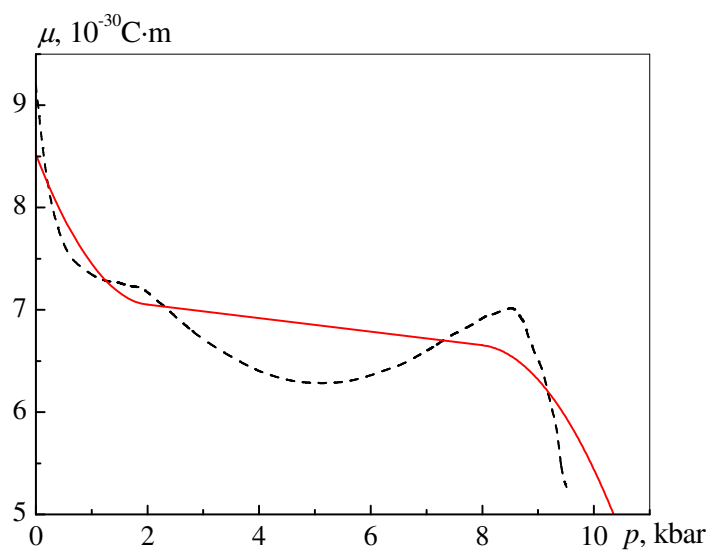

Fig. 6. Dependence of the effective dipole moment on hydrostatic pressure at $T=292 \mathrm{~K}$ derived for parameters set 7 (dashed line) along with its approximation (2.4) (solid line).
Experimental data for baric dependencies of the polarization at $T=292 \mathrm{~K}$ along with calculation results derived basing on parameters set 7 and effective dipole moment approximated by (2.4) are presented in figure 5 . As figure shows the accordance is satisfactory.

On the basis of approximation (2.4) and dependence (2.1) for $\mu(T)$ we propose the following expression for temperature and pressure dependent effective dipole moment:

$$
\mu(T, p)=\mu_{0}(p)+k(T-292)
$$

where $k=-0.022 \cdot 10^{-30}(\mathrm{C} \cdot \mathrm{m}) / \mathrm{K}$, and $\mu_{0}(p)$ is defined by (2.4). Basing on the derived theory model parameters (parameters set 7 in (2.3) and effective dipole moment (2.5)) we calculated other thermodynamic characteristics of the Rochelle salt at different values of hydrostatic pressure.

Out of thermodynamic characteristics of the Rochelle salt experimentally studied at different hydrostatic pressures we know only data for temperature dependencies of longitudinal static dielectric permittivity of a free crystal $\varepsilon_{11}$ derived in the works $[12,15]$. However, these dependencies are somewhat strange, as they do not have 
singularities at phase transition points (in particular at lower transition point at $p=0 \mathrm{kbar}$ ), which contradicts experimental data of other works, for example [23,24].

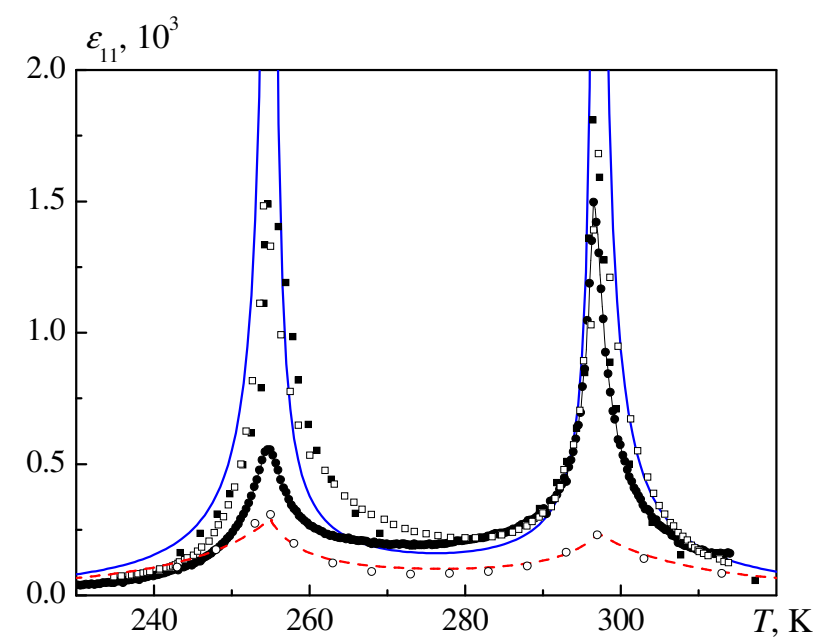

Fig. 7. Temperature dependencies of the static dielectric permittivity $\varepsilon_{11}$ in the absence of hydrostatic pressure. Points: •, $\square$, are the experimental data derived for a free crystal in [15], [23] and [12] respectively, o - the experimental data derived for a clamped crystal in [25]. Lines are the calculation results derived in [16] for a free crystal (solid) and clamped (dashed) crystal.

One should notice some similarity in the temperature behaviour of dielectric permittivities measured in works $[12,15]$ at increasing hydrostatic pressure. In these works lower peaks of dielectric permittivity were derived decreasing with applying hydrostatic pressure while the height of upper peaks remain almost unchanged. Such similarity is clear to be not accidental, and we shall try and explain the reasons for such behaviour herein below. We should note that the model, proposed to describe Rochelle salt physical properties, reveals singularities of dielectric permittivity of a free crystal at phase transition points. Samara [12] explains absence of singularities on the dielectric permittivity at the low phase transition point by the peculiarity of experiment, causing partial clamping of the crystal. In particular, Samara attributes the decrease of lower dielectric permittivity peaks height subjected to an increase of the applied hydrostatic pressure to the increase of clamping which is in turn caused by an increase of transmitting medium viscosity subjected to applying pressure. At the same time, we can explain experimentally derived lower dielectric permittivity peaks heights at the lower point of phase transition as compared to the peaks heights at the upper point of phase transition by means of increasing of transmitting medium viscosity subjected to temperature decrease. The derived experimental results let us suggest that the crystal in the neighbourhood of lower phase transition temperature was partially clamped whereas in the neighbourhood of upper phase transition temperature it was free. Therefore, we face the fact that it is impossible to agree theory and experimental data of works $[12,15]$.



Fig. 8. Experimental data for temperature dependencies of dielectric permittivities $\varepsilon_{11}$ measured at hydrostatic pressures $p=0.0,2.2,3.0,4.1,5.0$ kbar derived in [12] along with the corresponding calculation results conducted for a free crystal at $p=0.00,1.95,2.83,3.80,4.85$ kbar.

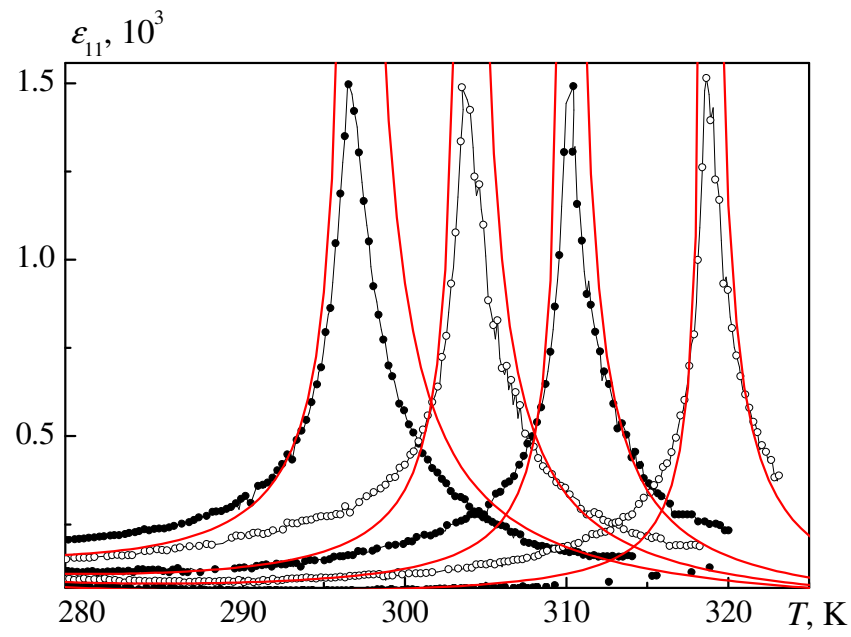

Fig. 9. Experimental data for temperature dependencies of dielectric permittivities $\varepsilon_{11}$ measured at hydrostatic pressures $p=0.0,0.5,1.2,2.0 \mathrm{kbar}$ derived in [15] along with the corresponding calculation results conducted for a free crystal at $p=0.00,0.57,1.10,1.85 \mathrm{kbar}$.

Figure 7 presents static dielectric permittivity ( $p=$ 0 kbar) measured in works $[12,15]$, permittivity of a free crystal [23], permittivity of a clamped crystal [25] and thes characteristics theoretically calculated in work [16]. As the figure shows, experimental data of work [15] are in poor agreement both with the data of work [23] and those of work [25]. However, work [12] results are in good agreement with results of work [23] derived for free crystal. The assumption about a partial crystal clamping in 
the experiment of work [15] in the vicinity of low transition point is supported by the height of dielectric permittivity peak in the low phase transition point, derived in this work: it is higher than the corresponding point of the clamped crystal and it is lower than the corresponding point of the free crystal ${ }^{1}$.
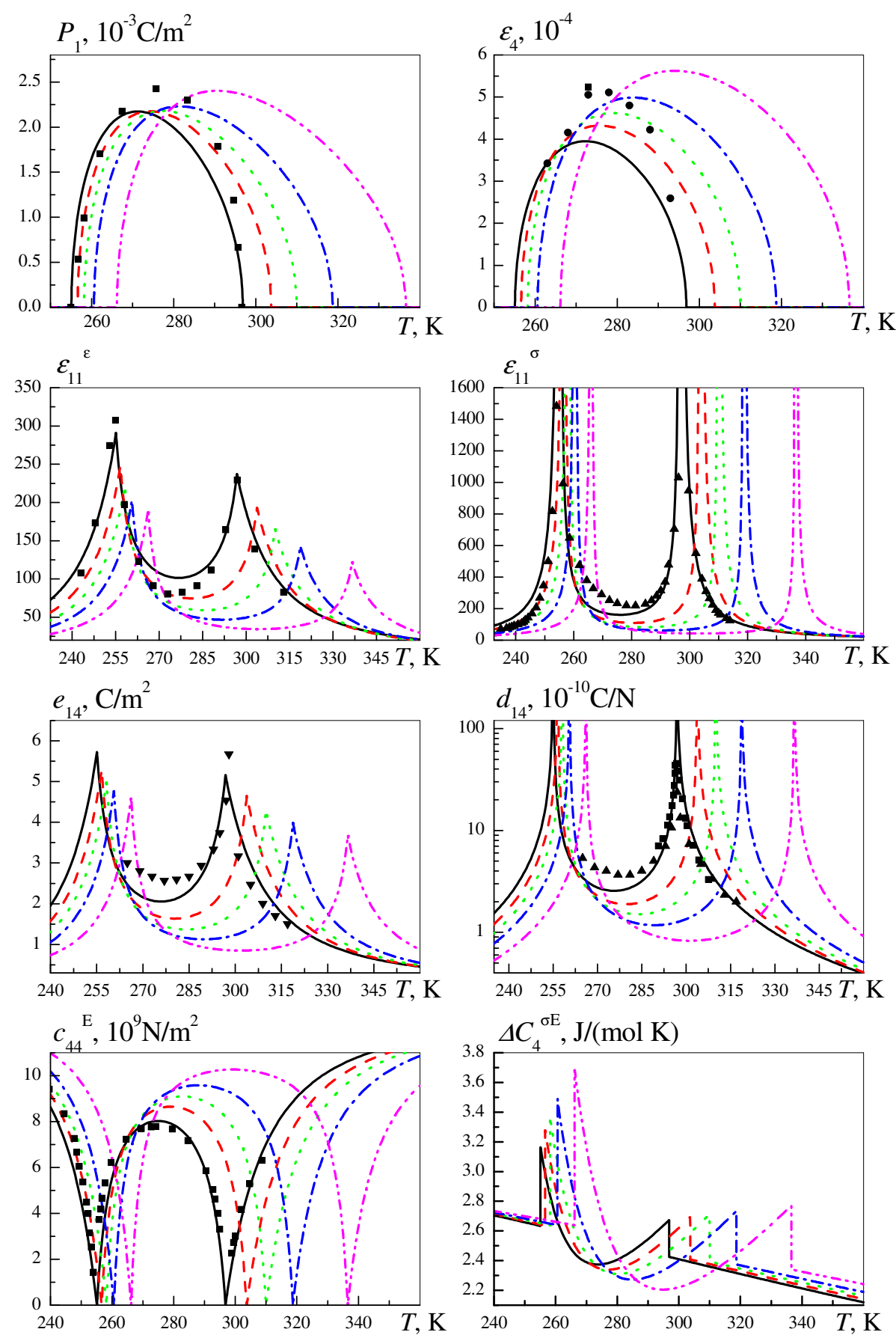

Fig. 10. Theoretically calculated physical characteristics of the Rochelle salt. Solid, dashed, dash-dotted, dash-dot-dotted lines are the results of calculations at $p=0.00,0.57,1.10,1.85,3.43 \mathrm{kbar}$ respectively. Experimental data $(p=0.00 \mathrm{kbar})$ $P_{1}(T): \boldsymbol{\square}-[26], \varepsilon_{4}(T): \mathbf{\square}-[27], \bullet-P_{1} d_{14} / \chi_{11}^{\sigma}[28], \varepsilon_{11}^{\varepsilon}(T): \boldsymbol{\square}-[25], \varepsilon_{11}^{\sigma}(T): \boldsymbol{\Delta}-[23], e_{14}(T): \boldsymbol{\nabla}-[29], d_{14}(T): \mathbf{\square}-[29]$, $\boldsymbol{\Delta}-[30], c_{44}^{\mathrm{E}}: \mathbf{\square}-[31]$.

\footnotetext{
${ }^{1}$ However, perhaps, it is not partial crystal clamping that causes "abnormal" dielectric permittivity behaviour measured in experiments conducted in $[12,15]$, but rather impurities and structure defects which may smoothen singularities at phase transition points. Yet, in this case, it is still not quite clear, why the effect of the latter becomes stronger in the vicinity of low transition point.
} 
We restricted our comparing of theoretical and experimental (works $[12,15]$ ) dependencies of dielectric permittivity of a free crystal at different hydrostatic pressures by a high temperature region (temperatures, close to the upper phase transition temperatures) since, as noted above, we consider a crystal to be partially clamped in the low temperatures region. The calculations were carried out at hydrostatic pressure values somewhat differently from the corresponding experimental values. Changing value of pressure we adjusted the positions of peaks in theoretically calculated dielectric permittivity, so that they agree with the corresponding peaks derived experimentally. That is to say, by changing the value of pressure we agree experimental and theoretically calculated phase transitions temperatures. So, if experiment in [12] was conducted at $p=0.0,2.2,3.0,4.1,5.0 \mathrm{kbar}$, then calculations were conducted at $p=0.00,1.95,2.83,3.80,4.85$ kbar respectively; if experiment in [15] was conducted at $p=$ $0.0,0.5,1.2,2.0 \mathrm{kbar}$, then calculations were conducted at $p=0.00,0.57,1.10,1.85$ kbar, respectively. Experimental data for temperature dependencies of dielectric permittivities at different hydrostatic pressures as in works $[12,15]$ along with the results of calculations derived for a free crystal are presented in figures 8, 9 . As the figures show, for both experiments agreement is satisfactory.

Figure 10 presents theoretically calculated physical characteristics of the Rochelle salt (spontaneous polarization $P_{1}$, spontaneous deformation $\varepsilon_{4}$, longitudinal static dielectric permittivities of a free crystal and clamped crystal $\varepsilon_{11}^{\sigma}, \varepsilon_{11}^{\varepsilon}$, the coefficient of the piezoelectric stress $e_{14}=\left(\partial P_{1} / \partial \varepsilon_{4}\right)_{\mathrm{E}_{1}}$, the coefficient of the piezoelectric strain $d_{14}=\left(\partial P_{1} / \partial \sigma_{4}\right)_{\mathrm{E}_{1}}$, the elastic constant at constant field $c_{44}^{\mathrm{E}}$, molar specific heat of the quasispin subsystem $\Delta C_{4}^{\sigma \mathrm{E}}$ ) at hydrostatic pressures $p=$ $0.00,0.57,1.10,1.85,3.43$ kbar. Along with theoretical results the experimental data at zero pressure were presented.

As one can see from the figure, saturation polarization increases with increasing hydrostatic pressure. It happens in spite of decreasing effective dipole moment. The agreement of this result with experimental data could serve as a criterion for applicability of the proposed theory to describe the effect of hydrostatic pressure on physical properties of the Rs. By now there has been no experiment conducted to define polarization dependency on temperature at hydrostatic pressures other than zero.

Similarly, spontaneous deformation increases with the increasing of hydrostatic pressure. Also, one can notice decreasing of the low dielectric permittivity peak of a clamped crystal subjected to an increase of hydrostatic pressure similarly to the behaviour of dielectric permittivity experimentally derived in works $[12,15]$. This fact supports the assumption that the crystal, used in experiment of these works in the vicinity of lower phase transition points, indeed was partially clamped. The specific heat peak in the low phase transition point increases with applying hydrostatic pressure, while peak in the upper phase transition point remains unchanged. The be- haviour of the physical characteristics, as those described above, remains similar for a higher hydrostatic pressures of at least up to $8 \mathrm{kbar}$.

\section{CONCLUSIONS}

This work offers a simplified approach to the description of the hydrostatic pressure effect on the physical properties of the Rochelle salt. According to this approach lattice strain, caused by applying hydrostatic pressure, just alters interaction constants of the Hamiltonian without altering its structure. A more consistent theory should take into account the interaction of the lattice strain with pseudospin system. Approach of this work may help to construct this more consistent theory.

Within the framework of a simplified approach it was obtained that only increasing dependencies of the model theory parameters $\tilde{J}_{0}(p), \tilde{K}_{0}(p), \tilde{\Delta}(p)$ on hydrostatic pressure allow us to derive correct baric dependencies of the phase transition temperatures of the Rochelle salt. Out of several parameters sets, linearly dependent on pressure, we choose one, which gives the best description of the change of phase transition temperature, induced by hydrostatic pressure.

Having appropriately defined the effective dipole moment we achieved good agreement between theory and experiment for dependency of polarization on the hydrostatic pressure at room temperature. Derived results show effective dipole moment to be decreasing with applying hydrostatic pressure. A poor agreement between theory and experiment for longitudinal static dielectric permittivity of a free crystal, in our opinion, results from some fallacy of the experiment.

Derived decreasing of the effective dipole moment subject to increasing hydrostatic pressure is quite expected as application of hydrostatic pressure results in the compressing of crystal elementary cell and shortening of distances between ions in the molecule. The shorter distances between ions in the molecule, the smaller dipole moment occurs in it. The derived dependence of effective dipole moment on the hydrostatic pressure agrees with the results of work [2] for KDP crystal. In this work effective dipole moment was modelled under the assumption of being dependent on the applied pressure only through a dependence of lattice constants on pressure. This simple model provides satisfactory agreement between theory and experiment for saturation polarization of KDP at different values of the applied hydrostatic pressure. However, probably, besides the described above mechanism of change of the effective dipole moment subjected to applying pressure, there exists one other, related to mutual shifting of the ions, mechanism. The complex dependence $\mu(p)$ derived for Rochelle salt in the present work supports this assertion. Having experimentally studied what particular changes in crystal structure are induced by hydrostatic pressure one can find out whether the derived herein dependence of the effective dipole moment on hydrostatic pressure agrees with real changes in crystal structure or not. 
Thermodynamic characteristics of the Rochelle salt were calculated basing on the derived model theory parameters. The calculation results for polarization were used as a ground on which the conformity criterion of the developed approach to the description of the hydrostatic pressure effect on the Rochelle salt was proposed. Based on the calculation results for permittivity of a free and clamped crystals the discrepancies in experiment data for these characteristics derived by different researchers were explained.
[1] I. V. Stasyuk, I. N. Biletskii, Bull. Acad. Sci. USSR, Phys. Ser. (Engl. Transl.) 4, (1983).

[2] I. V. Stasyuk, R. R. Levitskii, A. P. Moina, Phys. Rev. B 59, 8530 (1999).

[3] R. O. Piltz, M. I. McMahon, R. J. Nelmes, Ferroelectrics 108, 271 (1990).

[4] I. V. Stasyuk, R. R. Levitskii, I. R. Zachek, A. P. Moina, Phys. Rev. B 62, 6198 (2000).

[5] I. V. Stasyuk, I. N. Biletskii, O. N. Styagar Ukr. Phys. J. 31, 567(1986) (in Ukrainian).

[6] I. V. Stasyuk, R. R. Levitskii, I. R. Zachek, A. S. Duda, Condens. Matter Phys. 4, 553 (2001).

[7] N. Yasuda, M. Okamoto, H. Shimizu, S. Fujimoto, K. Yoshino, and Y. Inuishi, Phys. Rev. Lett. 41, 1311 (1978).

[8] N. Yasuda, S. Fujimoto, M. Okamoto, H. Shimizu, K. Yoshino, and Y. Inuishi, Phys. Rev. B 20, 2755 (1979).

[9] R. Blinc, F. C. Sa Barreto, J. Chem. Phys. 72, 6031 (1980).

[10] I. V. Stasyuk, R. R. Levitskii, I. R. Zachek, Ya. J. Schur, I. V. Kutny, Ye. V. Mits, preprint ICMP-91-4P (in Russian), Lviv, 1991.

[11] D. Bancroft, Phys. Rev. 53, 587 (1938).

[12] G. A. Samara, J. Phys. Chem. Solids 26, 121 (1965).

[13] G. A. Samara, J. Phys. Chem. Solids 29, 870 (1968).

[14] K. Mori, M. Hayashi, J. Phys. Soc. Jpn 33, 1396 (1972).

[15] A. G. Slivka, V. M. Kedyulich, R. R. Levitskii, A. P. Moina, M. O. Romanyuk, A. M. Guivan, Condens. Matter Phys. 8, 623 (2005).
[16] R. R. Levitskii, I. R. Zachek, T. M. Verkholyak, and A. P. Moina, Phys. Rev. B 67, 174112 (2003).

[17] A. P. Moina, R. R. Levitskii, I. R. Zachek, Phys. Rev. B 71, 134108 (2005).

[18] R. R. Levitskii, I. R. Zachek, T. M. Verkholyak, A. P. Moina, Condens. Matter Phys. 6, 261 (2003).

[19] D. Berlincourt, D. R. Currar, H. Jaffe, Physical Acoustics, edited by P. W. Mason (Academic Press, New York, 1964).

[20] W. J. Bronowska, J. Appl. Crystallogr. 14, 203 (1981).

[21] W. P. Mason, Piezoelectric Crystals and Their Application to Ultrasonics (Van Nostrand, New York, 1950).

[22] Yu. I. Dublenych, preprint ICMP-02-15U (in Ukrainian), Lviv, 2002.

[23] W. Taylor, D. J. Lockwood, H. J. Labbe, J. Phys. C.: Solid State Phys. 17, 3685 (1984).

[24] A. V. Shyl'nikov et al., Kristallografiya 31, 326 (1986).

[25] F. Sandy, R. V. Jones, Phys. Rev. 168, 481 (1968).

[26] J. Hablützel, Helv. Phys. Acta 12, 489 (1939).

[27] A. R. Ubbelohde, I. Woodward, Proc. Roy. Soc. 185, 448 (1946).

[28] W. G. Cady, Piezoelectricity; an introduction to the theory and application ofelectromechanical phenomena in crystals (McGraw Hill BookCompany, Inc., New York, 1946).

[29] L. Gutin, Zh. Eksp. Teor. Fiz. 15, 199 (1945).

[30] H. Beige, A. Kühnel, Phys. Status Solidi A 84, 433 (1984).

[31] O. Yu. Serdobolskaya, Solid State Phys. 38, 1529 (1996).

\title{
ДОСЛІДЖЕННЯ ВПЛИВУ ГІДРОСТАТИЧНОГО ТИСКУ НА ТЕРМОДИНАМІЧНІ ВЛАСТИВОСТІ КРИСТАЛУ СЕГНЕТОВОЇ СОЛІ
}

\author{
Р. Р. Левицький ${ }^{1}$, А. П. Моїна ${ }^{1}$, А. Я. Андрусик ${ }^{1}$, А. Г. Сливка ${ }^{2}$, В. М. Кедюлич ${ }^{2}$ \\ ${ }^{1}$ Інститут фізики конденсованих систем НАН України, вул. Свєнціиъкого, 1, Львів, UА-79011, Україна \\ ${ }^{2}$ Ужсгородсъкий національний університет, вул. Підгірна, 48, Ужгород, UA-88000, Украӥна
}

У межах двопідграткової моделі Міцуї, що враховує п'єзоелектричну взаємодію, теоретично вивчено вплив гідростатичного тиску на термодинамічні властивості селнетової солі. Для дослідження запропоновано підхід, який базується на припущенні, що деформація кристалічної гратки, спричинена дією гідростатичного тиску, лише перенормовує константи взаємодії в гамільтоніані, не змінюючи його структури. Відповідно до цього припущення, обмежившись лінійними розкладами модельних параметрів теорії щодо тиску, досліджено поведінку моделі від прикладеного гідростатичного тиску. Обчислення зроблено в наближенні молекулярного поля. Одержано, що лише зростаючі модельні параметри теорії з гідростатичним тиском можуть описати спостережувані залежності температур фазового переходу від тиску.

Вибір параметрів теорії контролювали фазовою діаграмою: точка параметрів теорії, що відповідає сегнетовій солі при певному значенні гідростатичного тиску, повинна бути в ділянці на фазовій діаграмі, у якій відбувається послідовність фазових переходів, спостережувана на експерименті. Із дев'яти одержаних наборів параметрів теорії вибрано один, який забезпечує найменше середньоквадратичне відхилення теоретично розрахованих баричних залежностей температур фазових переходів від відповідних експериментальних залежностей. 
THE STUDY OF THE HYDROSTATIC PRESSURE EFFECT ON THE THERMODYNAMIC PROPERTIES...

Ефективний дипольний момент вибрано з умови узгодження теорії та експерименту для залежності поляризації від гідростатичного тиску при кімнатній температурі. Для ефективного дипольного моменту одержано спадну з гідростатичним тиском залежність.

Із отриманими модельними параметрами теорії та ефективним дипольним моментом розраховано температурні залежності пружних, діелектричних, п'єзоелектричних та теплових характеристик сегнетової солі при різних значеннях гідростатичного тиску й порівняно їх із наявними експериментальними даними. На основі порівняння одержаних результатів розрахунку для проникностей вільного та затиснутого кристала запропоновано пояснення розбіжностей в експериментальних даних, які отримали різні дослідники для статичної діелектричної проникності вільного кристала. Передбачено, що поляризація насичення зростатиме із прикладанням гідростатичного тиску. Цей установлений теоретично факт запропоновано використати як критерій адекватності розробленого підходу до опису впливу гідростатичного тиску на фізичні властивості кристала сегнетової солі. 